\title{
Synthesis, Characterization and Antibacterial Activity of Biologically Important Vanillin Related Hydrazone Derivatives
}

\author{
Thiyagarajan Govindasami ${ }^{1}$, Anjana Pandey ${ }^{2}$, Nithya Palanivelu ${ }^{3}$, Ashutosh Pandey ${ }^{*}$ \\ ${ }^{1}$ Department of Chemistry, Motilal Nehru National Institute of Technology, Allahabad, India \\ ${ }^{2}$ Department of Biotechnology, University of Allahabad, Allahabad, India \\ ${ }^{3}$ Department of Chemistry, BharathiarUniversity, Coimbatore, India \\ E-mail: apandey70@yahoo.com \\ Received June 3, 2011; revised July 27, 2011; accepted August 5, 2011
}

\begin{abstract}
Hydrazone derivatives of vanillin are found to possess anti-bacterial activities. Based on higher bio-activity of hydrazones, new hydrazone derivatives were synthesized from Piperdine-4-carboxylicacid methyl ester (1). The compounds 1-pyrimidine-2-yl piperidine-4-carboxylicacid(4-hydroxy-3-methoxy benzylidine)-hydrazide (10), 1-pyrimidine-2-yl piperidine-4-carboxylicacid (3,4-dimethoxy benzylidine) hydrazide (11), 1-pyrimidine-2-yl piperidine-4-carboxylicacid(4-butoxy-3-methoxy benzylidine)-hydrazide (12), 1-pyrimidine-2-yl piperidine-4-carboxylicacid(3-methoxy-4(2-methoxy ethoxy) benzylidine)-hydrazide (13) were synthesized, purified and characterized by ${ }^{1} \mathrm{HNMR},{ }^{13} \mathrm{CNMR}$, LCMS, FT-IR and HPLC techniques. The synthesized hydrazone derivatives were further checked for anti-bacterial activities by paper disc diffusion method against Pseudomonas aeruginosa and Staphylococcus aureus bacterial strains.
\end{abstract}

Keywords: Antibiotics, Fractional Crystallization, Hydrazones, Coupling Reaction

\section{Introduction}

Earlier, by Quantitative Structure Activity Relationship (QSAR) studies, most of the rifamycin derivatives were found to be biologically active compared to other compounds [1]. For example, the hydrazones obtained from 3 -formyl rifamycin and $\mathrm{N}$-amino-N-methyl piperazine derivatives were found to be biologically active and tested for oral treatment of infections in animals [2]. Recently, a lot of biologically important hydrazone derivatives with a number of functional groups have been synthesized from aromatic and aliphatic compounds [3]. Hydrazone derivatives are molecules containing highly reactive azomethine group $(\mathrm{CO}-\mathrm{NH}-\mathrm{N}=\mathrm{CH})$ and thus useful in new drug development [4]. Also, these are found to possess anti-microbial [5-7], anti-mycobacterial [8], anti-convulsant [9], analgesic [10], anti-inflammatory [11,12], anti-platelet [13], anti-tubercular [14-16] and anti-tumoral [17-19] activities. Diflunisal hydrazones were also prepared as possible dual acting antimicrobial and anti-tuberculosis agents with anti-inflammatory properties [20]. Moreover, hydrazones has been recently established as a good precursor for one-pot synthesis of C-4 functionalized 1,2,3,4-tetrahydro quinolones containing a quaternary stereo center [21]. Due to the growth of population and changes in climatic conditions several new diseases are likely to affect the human beings. So, there is a continuous need for the synthesis of new biologically active organic compounds by using a fast and efficient approach which may act as potential antimicrobial agents. Based on the higher bio-reactivity of hydrazones, we have synthesized novel hydrazones (10-13) from Piperdine-4-carboxylic acid methyl ester (1) coupled with 2-chloro pyrimidine (2) along with other vanillin derivatives (6-9). The anti-bacterial studies were effectively done for newly synthesized hydrazones by standard disc diffusion method [22] with different concentrations.

\section{Results and Discussion}

\subsection{Synthesis}

Earlier studies on pyrimidine shows, that heterocyclic 
compounds containing pyrimidine moiety shows various biological activities. Therefore, we were tempted to synthesize vanillin related hydrazones with a pyrimidine moiety. A series of vanillin related hydrazones are synthesized and their purity is checked by thin layer chromatography (TLC) and HPLC techniques. All the synthesized hydrazones structures are characterized by ${ }^{1} \mathrm{HNMR}$ along with ${ }^{13} \mathrm{CNMR}$; LC-MS and FT-IR spectral techniques. There are three different types of coupling reactions taking place in syntheses of the hydrazone derivatives. In step-1, compound 1 is coupled with 2 to form 3 by "chloro-amine" coupling. In step- 2 the product 3 was reacted with 4 to form 5 by "ester-amine" condensation. In step-3, vanillin derivatives (6-9) react with 5 to form hydrazone derivatives $\mathbf{( 1 0 - 1 3 )}$ by "aldehyde-amine" coupling. From the ${ }^{1} \mathrm{H}$ NMR spectra, the structures of the synthesized compounds (10-13) were confirmed on the basis of the fact that the aldehydic proton (which was visible at $\delta 10.55)$ in the starting compound 6 disappeared, and a new singlet due to the azomethine $(\mathrm{CH}=\mathrm{N})$ group appeared at $\delta$ values between $8.06-8.11 \mathrm{ppm}$ in all the compounds. The $\mathrm{CONH}$ protons appearing as singlets resonated at $\delta$ values between 11.20 and 11.25 ppm. Furthermore, the protons of $\mathrm{CONH}$ and $\mathrm{CH}=\mathrm{N}$ exhibited two separate signals in 1HNMR spectra in between 11.20 - 11.25 ppm and 8.06 - 8.11 ppm respectively due to the nitrogen inversion, which is shown in

\section{Figure 1.}

The three $-\mathrm{CH}$ protons of the pyrimidine $(\mathrm{pm})$ were centered at $\delta$ value $8.35 \mathrm{ppm}$ as doublets by integrating in two proton and at $\delta$ value $6.60 \mathrm{ppm}$ as triplets by integrating in one proton.

In the ${ }^{13} \mathrm{CNMR}$ spectra of 6 the carbon signal due to (-CHO), was observed at $\delta 188.97 \mathrm{ppm}$. However in products 10-13 this signal was found to be absent and a new signal at $\delta$ values between 150.04 and 151.04 ppm arose due to the presence of $\mathrm{CH}=\mathrm{N}$ in compounds (10-13).

The carbon signal of $\mathrm{C}=\mathrm{O}$ group appeared at $\delta$ values between 175.8 and $176.2 \mathrm{ppm}$. The molecular mass of the synthesized compounds were recorded by LC-MS techniques, which was registered in positive ion $(+\mathrm{M})$ mode. The FT-IR spectra of compounds (10-13) showed absorption bands at $1652-1655 \mathrm{~cm}^{-1}$ due to the presence of $\mathrm{C}=\mathrm{O}$ functional group, while the bands observed at $1582-1586 \mathrm{~cm}^{-1}$ corresponded with $\mathrm{C}=\mathrm{N}$ linkage and $3280-3413 \mathrm{~cm}^{-1}$ observed due to the $-\mathrm{NH}$ group. The absorption peak at $2845-2867 \mathrm{~cm}^{-1}$ was due to the $\mathrm{CH}$ linkage and the band appearing at $3845.4 \mathrm{~cm}^{-1}$ in the IR spectrum of the compound (10) represented $\mathrm{OH}$ group. The synthetic conditions and melting points of the newly synthesized compounds are summarized in Table 1.

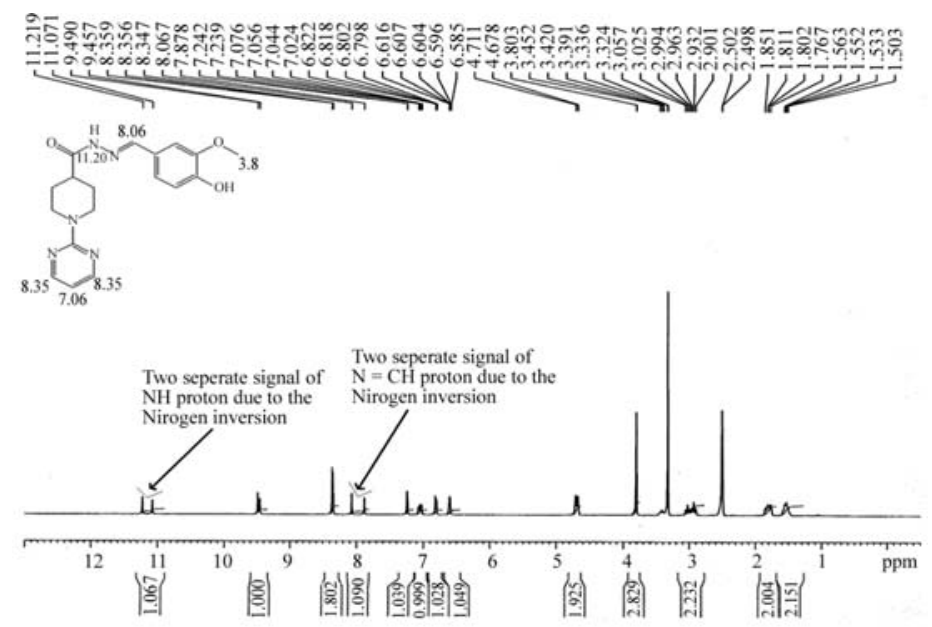

Figure 1. Nitrogen inversion of compound 10.

Table 1. Reaction data of newly synthesized compounds

\begin{tabular}{|c|c|c|c|c|}
\hline Compound & Condition & Purification method & HPLC purity & LCMS $(+\mathrm{M})$ \\
\hline 3 & Acetonitrile, $85^{\circ} \mathrm{C}$, Reflux, $6 \mathrm{~h}$ & Column, $10 \%$ Ethyl acetate:Hexane & 98.7 & 222.3 \\
\hline 5 & Methanol, $90^{\circ} \mathrm{C}$, Reflux, $5 \mathrm{~h}$ & Crystallization, Methonol, $10 \mathrm{~mL}$ & 99.6 & 222.6 \\
\hline 10 & Ethanol, $70^{\circ} \mathrm{C}$, Reflux, $8 \mathrm{~h}$ & FC, Ethyl acetate:diethyl ether $(5: 10) \mathrm{mL}$ & 99.8 & 355.8 \\
\hline 11 & Ethanol, $70^{\circ} \mathrm{C}$, Reflux, $8 \mathrm{~h}$ & FC,Dichloro methane:Hexane $(3: 8) \mathrm{mL}$ & 99.6 & 370.2 \\
\hline 12 & Ethanol, $70^{\circ} \mathrm{C}$, Reflux, $8 \mathrm{~h}$ & FC, Dichloro methane:di ethyl ether $(2: 5) \mathrm{mL}$ & 96.8 & 412.8 \\
\hline 13 & Ethanol, $70^{\circ} \mathrm{C}$, Reflux, $8 \mathrm{~h}$ & FC, Ethyl acetate:Diethyl ether (5:6:4) mL & 97.8 & 414.8 \\
\hline
\end{tabular}

FC- fractional crystallization. 


\subsection{Antibacterial Activity}

The anti-bacterial results showed that some of the compounds were active against both Gram-positive S. aureus and Gram-negative P. aeruginosa bacteria. Among the tested solutions (10-13), the compounds (12) and (13) showed good antibacterial activity against the test organisms and 11 had moderate effective against $S$. aureus and less effective against $P$. aeruginosa. The compound 10 had no anti-bacterial activity against $P$. aeruginosa and lowest activity against $S$. aureus. It was observed that maximum antibacterial activity was shown by compounds containing the butoxy, methoxy and methylethoxy group with highly reactive azomethine (-NH-N= $\mathrm{CH}-$ ) group. On the other hand, compared to the standard antibacterial drugs namely, Ciprofloxacin and Cefaclor our synthesized hydrazones were having moderate activeity against test organisms. The obtained results of antibacterial activity have been summarized in Table 2 .

\section{Experimental}

All synthetic manipulations were conducted in the dry and nitrogen atmosphere. Solvents for synthesis were reagent grade and dried by standard procedures [23]. The starting materials are such as (1), (2), Hydrazine hydrate (4), Vanillin (6) and Veratraldehyde (7) were obtained from Sigma-Aldrich chemicals and acetone, methanol, ethanol, acetonitrile and dichloromethane, which were obtained from SRL Chemical Limited, India. The intermediate vanillin derivatives such as 4-butoxy-3-methoxy benzaldehyde (8) and 3-methoxy-4-(2-methoxy-ethoxy) benzaldehyde (9) were prepared by typical procedures $[24,25]$. Melting points of as synthesized compounds were determined with open capillary tube on a Gallenkamp melting point apparatus. The ${ }^{1} \mathrm{H}$ and ${ }^{13} \mathrm{CNMR}$ were recorded on a Bruker Avance-III, $300 \mathrm{MHz}$ and 400 MHz. Liquid chromatography mass spectra (LCMS) were run on "LCMS-Agilent Technologies-1200 Series" and purity was checked by "HPLC — Agilent Technogies-1200 Series". IR spectra were recorded by "FTIR Nicolet 6700" spectrometer. All compounds were routinely checked by TLC on silica gel G plates using petroleum ether/ethyl acetate $(7: 3 ; 6: 4 ; 5: 5$ by $\mathrm{V} / \mathrm{V})$ as solvent system and the developed plates were visualized by UV light, iodine vapour and $\mathrm{KMnO}_{4}$ solution. The detailed scheme of synthesis has been shown in Scheme 1. The anti-bacterial studies performed in Center for Biotechnology, University of Allahabad, India.

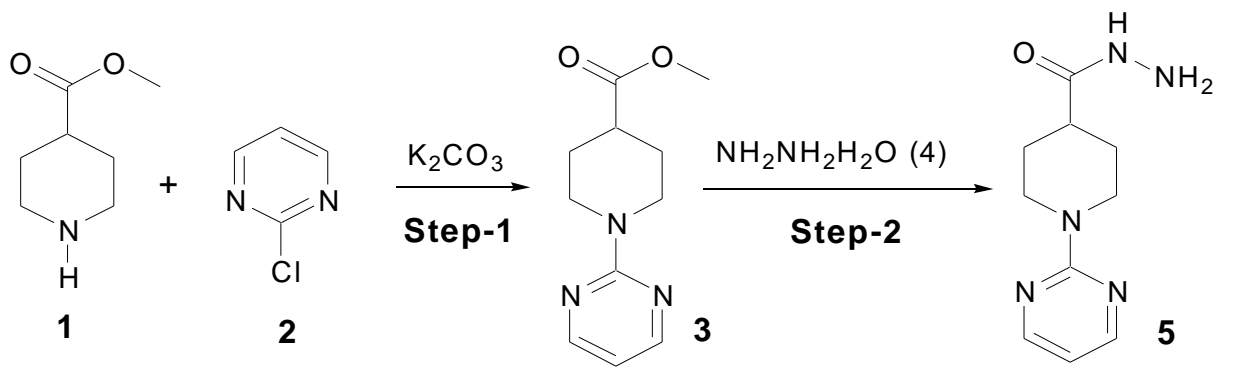

1 - Piperdine-4-carboxylic acid methyl ester

2 - 2-Chloro pyrimidine

3 - 1-pyrimidine-2-yl-piperdine-4-carboxylic acid methyl ester

4 - Hydrazine hydrate

5 - 1-Pyrimidin-2-yl-piperidine-4-carboxylic acid hydrazide

6 - Vanillin

7 - Veratradehyde

8 - 4-Butoxy-3-methoxy-benzaldehyde

9 - 3-Methoxy-4-(2-methoxy-ethoxy) benzaldehyde

10 - 1-Pyrimidin-2-yl-piperidine-4-carboxylic acid (4-hydroxy-3-methoxy benzylidene) - hydrazide

11 - 1-Pyrimidin-2-yl-piperidine-4-carboxylicacid (3,4-dimethoxy benzylidene) hydrazide

12 - 1-Pyrimidin-2-yl-piperidine-4-carboxylic acid (4-butoxy-3-methoxy - benzylidene)-hydrazide

13 - 1-Pyrimidin-2-yl-piperidine-4-carboxylic acid [3-methoxy-4-(2-methoxy-ethoxy)-benzylidene] hydrazide
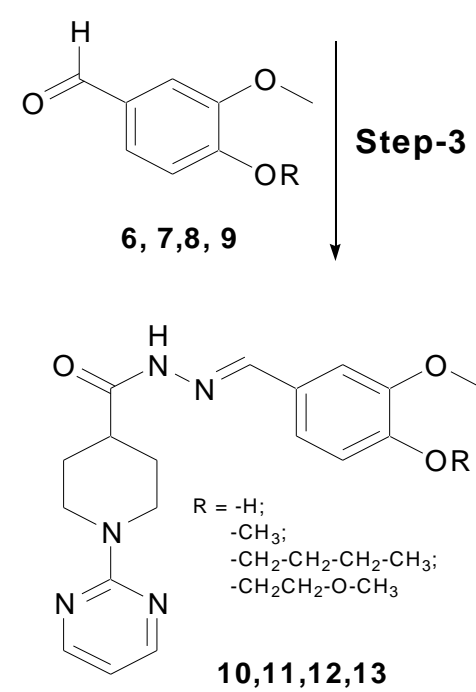

Scheme 1. Synthetic scheme of novel hydrazone derivatives. 
Table 2 .Antibacterial activity of novel hydrazones $\left(\mu \cdot \mathrm{gmL}^{-1}\right)$

\begin{tabular}{|c|c|c|c|c|c|c|c|c|c|c|}
\hline \multirow{2}{*}{$\begin{array}{l}\text { Compound } \\
\left(\mu \mathrm{g} \cdot \mathrm{mL}^{-1}\right)\end{array}$} & \multicolumn{5}{|c|}{ Staphylococcus aureus $(\mathrm{G}+)$} & \multicolumn{5}{|c|}{ Pseudomonas aeruginosa $(\mathrm{G}-)$} \\
\hline & 50 & 100 & 150 & 200 & 250 & 50 & 100 & 150 & 200 & 250 \\
\hline 10 & - & + & + & ++ & ++ & - & - & - & - & + \\
\hline 11 & + & + & ++ & +++ & +++ & - & - & + & ++ & ++ \\
\hline 12 & - & + & + & ++ & +++ & + & ++ & +++ & +++ & +++ \\
\hline 13 & + & ++ & +++ & +++ & +++ & ++ & ++ & +++ & +++ & ++++ \\
\hline DMSO & - & - & - & - & - & - & - & - & - & - \\
\hline Ciprofloxacin & ++ & +++ & ++++ & ++++ & +++++ & +++ & +++ & ++++ & ++++ & +++++ \\
\hline Cefoclor & +++ & +++ & ++++ & ++++ & +++++ & - & - & - & - & - \\
\hline
\end{tabular}

(-) No measurable activity; (+ )1 - $2 \mathrm{~mm} ;(++) 3$ - $5 \mathrm{~mm} ;(+++) 6-8 \mathrm{~mm} ;(++++) 9$ - $12 \mathrm{~mm} ;(+++++) 13-17 \mathrm{~mm}$

\subsection{Synthesis of 1-Pyrimidine-2-yl piperdine-4-carboxylicacid Methyl Ester (3)}

Methyl nipocotate [Piperdine-4-carboxylic acid methyl ester (1) $(1.3 \mathrm{~mL}, 8.7 \mathrm{mmol}, 1.0 \mathrm{eq}$.) $]$ and potassium carbonate $(1.2 \mathrm{~g}, 8.7 \mathrm{mmol}, 1.0 \mathrm{eq}$.) was added to a stirred solution of 2-Chloro pyrimidine (2) (1.0 g, $8.7 \mathrm{mmol}, 1.0$ eq) in dry acetonitrile $(10 \mathrm{~mL})$ under nitrogen atmosphere and refluxed at $85^{\circ} \mathrm{C}$ in a sealed tube for $10 \mathrm{hrs}$ followed by cooling to room temperature. The solvent was evaporated under high vacuum and the crude product was extracted with ethyl acetate $(3 \times 50 \mathrm{~mL})$. The organic layer was washed with water and brine, dried with sodium sulfate, filtered and concentrated under reduced pressure. The so obtained product was purified by column chromatography using $10 \%$ ethyl acetate in petroleum ether as eluant [Silica gel; Rf: 0.3 (Pet Ether:EA; 7:3)] to get the product (1-pyrimidine-2-yl-piperdine4-carboxylic acid (3)) as yellow liquid. $1.8 \mathrm{~g}$, Yield: $93 \%$. ${ }^{1} \mathrm{HNMR}:\left(\mathrm{CDCl}_{3}, 300 \mathrm{MHz}\right), \delta 8.30(\mathrm{~d}, 2 \mathrm{H}, J=2.6 \mathrm{~Hz}$, pm-N-CH), $6.47(\mathrm{t}, J=6.00 \mathrm{~Hz}, 1 \mathrm{H}, \mathrm{Pm}-\mathrm{CH}), 4.67$ and 3.08 (m, 4H, Py- $\left.\mathrm{NCH}_{2}\right), 3.70$ (s, 3H, $\left.-\mathrm{CH}_{3}\right), 2.61(\mathrm{~m}, 1 \mathrm{H}$, Py-CH), $2.0-1.70$ (m, 4H, - $\left.\mathrm{PyCH}_{2}\right) ;{ }^{13} \mathrm{CNMR}$ : $\left(\mathrm{CDCl}_{3}\right.$, $300 \mathrm{MHz}), \delta 175.2(1 \mathrm{C}, \mathrm{C}=\mathrm{O}), 161.5(1 \mathrm{C}, \mathrm{Pm}-\mathrm{N}-\mathrm{C}-\mathrm{N})$, 157.6 (2C, Pm-N-CH), 109.6 (1C, Pm-CH), 51.7 (1C, $\left.\mathrm{O}_{-} \mathrm{CH}_{3}\right), 43.1$ (2C, Py-N-CH $), 41.4$ (1C, Py-CH), 27.8 (2C, Py- $\mathrm{CH}_{2}$ ); LCMS: 221.3 (Calculated mass for $\mathrm{M}+$, 222.3); HPLC purity: $98.7 \%$.

\subsection{Synthesis of 1-Pyrimidin-2-yl piperidine-4-carboxylic Acid Hydrazide (5)}

To a dry RB flask the product (3) (1.0 g; $4.5 \mathrm{mmol}$; 1eq) was added to dry methanol $(10 \mathrm{ml})$ containing Hydrazine hydrate (4) $(1.3 \mathrm{ml} ; 27 \mathrm{mmol}$; 8eq.) under nitrogen atmosphere and refluxed to $95^{\circ} \mathrm{C}$ in sealed tube for $7 \mathrm{hrs}$. The reaction mixture cooled to room temperature, concentrate under reduced pressure. The crude was purified by crystallization, washed with petroleum ether and filtered. White solid, $0.96 \mathrm{~g}$ Yield: $96 \% .{ }^{1} \mathrm{HNMR}:\left(\mathrm{CDCl}_{3}\right.$,
$300 \mathrm{MHz}), \delta 9.02(\mathrm{~s}, 1 \mathrm{H},-\mathrm{NH}), 8.34$ (d, $2 \mathrm{H}, J=2.4 \mathrm{~Hz}$, Pm-N-CH), 6.60 (t, $J=7.20 \mathrm{~Hz}, 1 \mathrm{H}, \mathrm{Pm}-\mathrm{CH}), 4.66$ and 2.90 (m, 4H, Py-N-CH $)_{2}, 4.16$ (d, 2H, $J=2.8, \mathrm{NH}_{2}$ ), 2.38 (m, $1 \mathrm{H}, \mathrm{Py}-\mathrm{CH}), 1.68-1.50\left(\mathrm{~m}, 4 \mathrm{H}, \mathrm{Py}-\mathrm{CH}_{2}\right) ;{ }^{13} \mathrm{CNMR}$ : $\left(\mathrm{CDCl}_{3}, 300 \mathrm{MHz}\right), \delta 174.02(1 \mathrm{C}, \mathrm{C}=\mathrm{O}), 161.5(1 \mathrm{C}$, Pm-N-C-N), 158.3 (2C, Pm-N-CH), 110.2 (1C, Pm-CH), 43.3 (2C, Py-N-CH ${ }_{2}$ ), 39.9 (1C, Py-CH), 28.3 (2C, Py$\mathrm{CH}_{2}$ ); LC-MS: 221.16 (Calculated mass for $\mathrm{M}+, 222.6$ ); HPLC purity: $99.6 \%$.

\subsection{General Procedure for Synthesis of (10-13)}

Vanillin derivatives (6-9) (1.5eq.) were added to compound (5) (1eq.) separately in dry ethanol/acetic acid (5:1 $\mathrm{mL}$ ) under nitrogen atmosphere and the reaction mixtures were refluxed at $85^{\circ} \mathrm{C}$ in sealed tube for $8 \mathrm{hrs}$. These were slowly brought to room temperature and concentrated under reduced pressure. The crude products purified by fractional crystallization method by using ethyl acetate or dichloromethane along with petroleum ether or diethyl ether were filtered and dried under vacuum to give the corresponding products (10-13) as white solids.

\subsubsection{1-Pyrimidin-2-yl-piperidine-4-carboxylicacid (4-hydroxy-3-methoxy benzylidene)- Hydrazide (10)}

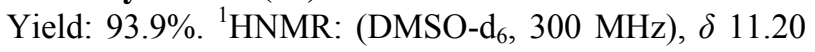
(s, 1H, -NH), 9.77 (s, $1 \mathrm{H},-\mathrm{OH}), 8.35$ (m, 2H, Pm-N-CH), $8.06(\mathrm{~s}, 1 \mathrm{H}, \mathrm{N}=\mathrm{CH}), 7.24,7.05$ and $6.80(3 \mathrm{H}, \mathrm{Ar}-\mathrm{CH})$, $6.60(\mathrm{~m}, 1 \mathrm{H}, \mathrm{Pm}-\mathrm{CH}), 4.70$ and $2.96\left(\mathrm{~m}, 4 \mathrm{H}, \mathrm{Py}-\mathrm{N}-\mathrm{CH}_{2}\right)$, 3.80 (s, $\left.3 \mathrm{H},-\mathrm{CH}_{3}\right), 1.81-1.55$ (m, 4H, $\left.\mathrm{Py}-\mathrm{CH}_{2}\right) ;{ }^{13} \mathrm{CNMR}$ : $\left(\mathrm{DMSO}_{6} \mathrm{~d}_{6}, 400 \mathrm{MHz}\right) \delta 170.2(1 \mathrm{C}, \mathrm{C}=\mathrm{O}), 161.1(1 \mathrm{C}$, $\mathrm{N}-\mathrm{C}-\mathrm{N}), 157.9$ (2C, Pm-N-CH), $151.4 \quad(1 \mathrm{C}, \mathrm{N}=\mathrm{CH})$, 146.7, 142.9, 125.7, 121.86, 115.3 and 108.8 (Ar-C), $109.8(1 \mathrm{C}, \mathrm{Pm}-\mathrm{CH}), 55.5\left(1 \mathrm{C}, \mathrm{O}-\mathrm{CH}_{3}\right), 42.8$ (2C, Py-N-CH ${ }_{2}$, 40.12 (1C, Py-CH), 27.8 (2C, Py-CH2); LC-MS: 354.4 (Calculated mass for M+, 355.8); FT-IR $\left(\mathrm{cm}^{-1}, \mathrm{KBr}\right): 3845.4(\mathrm{OH}), 3413.4(-\mathrm{NH}), 2854.7(-\mathrm{CH})$, $1662(\mathrm{C}=\mathrm{O}), 1583.1(\mathrm{C}=\mathrm{N})$; HPLC purity: $99.8 \%$; mp: $233.1^{\circ} \mathrm{C}-234.4^{\circ} \mathrm{C}$. 


\subsubsection{1-Pyrimidin-2-yl-piperidine-4-carboxylicacid (3,4-dimethoxy benzylidene) Hydrazide (11)}

Yield: 96\%. ${ }^{1} \mathrm{HNMR}$ : (DMSO-d 6 , $\left.300 \mathrm{MHz}\right), \delta 11.29$ (m, $1 \mathrm{H},-\mathrm{NH}), 8.35$ (d, $J=3.00 \mathrm{~Hz}, 2 \mathrm{H}, \mathrm{Pm}-\mathrm{N}-\mathrm{CH}), 8.11$ (s, $1 \mathrm{H}, \mathrm{N}=\mathrm{CH}), 7.27,7.16$ and $7.00(3 \mathrm{H}, \mathrm{Ar}-\mathrm{CH}), 6.60(\mathrm{~m}$, $1 \mathrm{H}, \mathrm{Pm}-\mathrm{CH}$ ), 4.70 and $2.96\left(\mathrm{~m}, 4 \mathrm{H}, \mathrm{Py}-\mathrm{N}-\mathrm{CH}_{2}\right), 3.80$ (s, $\left.6 \mathrm{H}, \mathrm{O}-\mathrm{CH}_{3}\right), 1.80-1.55\left(\mathrm{~m}, 4 \mathrm{H}, \mathrm{Py}-\mathrm{CH}_{2}\right) ;{ }^{13} \mathrm{CNMR}$ : $\left(\mathrm{DM}-\mathrm{SO}-\mathrm{d}_{6}, 300 \mathrm{MHz}\right), \delta 176.2(1 \mathrm{C}, \mathrm{C}=\mathrm{O}), 161.6(1 \mathrm{C}$, N-C-N), 158.4 (2C, Pm-N-CH), $151.0(1 \mathrm{C}, \mathrm{N}=\mathrm{CH})$, 150.8, 149.4, 127.5, 122.0 and 112.1 (Ar-C), 108.9 (1C, Pm-CH), 108.7 (Ar-C), 56.0 (2C, O-CH ${ }_{3}$, 43.4 (2C, Py-N$\left.\mathrm{CH}_{2}\right)$, 38.57 (1C, Py-CH), $28.3\left(2 \mathrm{C}, \mathrm{Py}_{-} \mathrm{CH}_{2}\right)$; LC-MS: 369.5 (Calculated mass for M+, 370.2); FT-IR $\left(\mathrm{cm}^{-1}, \mathrm{KBr}\right)$ : $3211.7(\mathrm{NH}), 2845.0(-\mathrm{CH}), 1652(\mathrm{C}=\mathrm{O}), 1582(\mathrm{C}=\mathrm{N})$; HPLC purity: $99.6 \%$; mp: $220.6^{\circ} \mathrm{C}-221.8^{\circ} \mathrm{C}$.

\subsubsection{1-Pyrimidin-2-yl-piperidine-4-carboxylicacid (4-Butoxy-3-methoxy) Benzylidene Hydrazide (12)}

Yield: 96.1\%. ${ }^{1} \mathrm{HNMR}:\left(\mathrm{DMSO}_{6} \mathrm{~d}_{6}, 300 \mathrm{MHz}\right), \delta 11.27$ (m, 1H, -NH), 8.34 (d, $J=4.50 \mathrm{~Hz}, 2 \mathrm{H}, \mathrm{Pm}-\mathrm{N}-\mathrm{CH}), 8.11$ (s, $1 \mathrm{H}, \mathrm{N}=\mathrm{CH}), 7.25,7.12$ and $6.97(3 \mathrm{H}, \mathrm{Ar}-\mathrm{CH}), 6.58(\mathrm{~m}$, $1 \mathrm{H}, \mathrm{Pm}-\mathrm{CH}), 4.68$ and $2.93\left(\mathrm{~m}, 4 \mathrm{H}, \mathrm{Py}-\mathrm{CH}_{2}\right), 3.97(\mathrm{~m}, 2 \mathrm{H}$, $\left.-\mathrm{OCH}_{2}\right), 3.77\left(\mathrm{~m}, 3 \mathrm{H},-\mathrm{OCH}_{3}\right), 1.71\left(\mathrm{~m}, 4 \mathrm{H},-\mathrm{CH}_{2}-\mathrm{CH}_{2}\right)$, $1.40\left(\mathrm{~m}, 4 \mathrm{H}, \mathrm{Py}-\mathrm{CH}_{2}\right), 0.92\left(\mathrm{~m}, 3 \mathrm{H},-\mathrm{CH}_{3}\right) .{ }^{13} \mathrm{CNMR}$ : $\left(\left(\mathrm{DMSO}_{-} \mathrm{d}_{6}, 300 \mathrm{MHz}\right), 176.0 \quad(1 \mathrm{C}, \mathrm{C}=\mathrm{O}), 161.6 \quad(1 \mathrm{C}\right.$, N-C-N), 158.4 (2C, Pm-N-CH), $150.4(1 \mathrm{C}, \mathrm{N}=\mathrm{CH}), 150.2$, 149.61, 127.4, 122.0, 113.1 and $112.1(\mathrm{Ar}-\mathrm{CH}), 110.2(1 \mathrm{C}$, Pm-CH), $68.3\left(1 \mathrm{C}, \mathrm{O}-\mathrm{CH}_{2}\right), 55.9\left(1 \mathrm{C}, \mathrm{O}-\mathrm{CH}_{3}\right), 43.4(2 \mathrm{C}$, Py-N-CH $)_{2}, 41.5(1 \mathrm{C}, \mathrm{Py}-\mathrm{CH}), 31.21\left(1 \mathrm{C}, \mathrm{CH}_{2}\right), 28.3(2 \mathrm{C}$, Py- $\left.\mathrm{CH}_{2}\right), 19.1\left(1 \mathrm{C}, \mathrm{CH}_{2}\right), 14.1\left(\left(1 \mathrm{C},-\mathrm{CH}_{3}\right)\right.$; LC-MS: 411.41 (Calculated mass for $\mathrm{M}+, 412.8)$; $\mathrm{IR}\left(\mathrm{cm}^{-1}, \mathrm{KBr}\right)$ : $3208.2(\mathrm{NH}), 2867.8(-\mathrm{CH}), 1655(\mathrm{C}=\mathrm{O}), 1583.9(\mathrm{C}=\mathrm{N})$; HPLC purity: $96.8 \%$. mp: $213.8^{\circ} \mathrm{C}-215.1^{\circ} \mathrm{C}$.

\subsubsection{1-Pyrimidin-2-yl-piperidine-4-carboxylicacid [3-methoxy-4-(2-methoxy ethoxy)-benzylidene Hydrazide (13)}

Yield: 78.1\%. ${ }^{1} \mathrm{HNMR}$ : (DMSO-d 6 , 300MHz), $\delta 11.25$ (m, $1 \mathrm{H},-\mathrm{NH}), 8.35$ (d, $J=2.8 \mathrm{~Hz}, 2 \mathrm{H}, \mathrm{Pm}-\mathrm{N}-\mathrm{CH}), 7.90$ $(\mathrm{s}, 1 \mathrm{H}, \mathrm{N}=\mathrm{CH}), 7.27,7.13$ and $6.99(3 \mathrm{H}, \mathrm{Ar}-\mathrm{CH}), 6.60$ (m, $1 \mathrm{H}, \mathrm{Pm}-\mathrm{CH}), 4.70$ and 2.95 (m, 4H, Py-N-CH $), 4.10$ (m, $\left.2 \mathrm{H},-\mathrm{O}-\mathrm{CH}_{2}\right), 3.65\left(\mathrm{~m}, 2 \mathrm{H},-\mathrm{CH}_{2}\right), 3.31$ (d, $J=7.8 \mathrm{~Hz}$, $\left.6 \mathrm{H},-\mathrm{O}-\mathrm{CH}_{3}\right), 1.81-1.52\left(\mathrm{~m}, 4 \mathrm{H}, \mathrm{Py}-\mathrm{CH}_{2}\right) ;{ }^{13} \mathrm{CNMR}$ : $\left(\mathrm{CDCl}_{3}, 400 \mathrm{MHz}\right), \delta 176.7(1 \mathrm{C}, \mathrm{C}=\mathrm{O}), 161.5(1 \mathrm{C}$, N-C-N), 157.7 (2C, Pm-N-CH), $150.3(1 \mathrm{C}, \mathrm{N}=\mathrm{CH})$, 149.84, 143.1, 127.0, 121.4 and 112.8 (Ar-C), 109.6 (1C, Pm-CH), 108.7 (Ar-C), 70.8, $68.3\left(\mathrm{CH}_{2}-\mathrm{O}-\mathrm{CH}_{2}\right), 59.29$ $\left(1 \mathrm{C}, \mathrm{O}-\mathrm{CH}_{3}\right), 55.8\left(1 \mathrm{C}, \mathrm{O}-\mathrm{CH}_{3}\right), 43.4\left(2 \mathrm{C}, \mathrm{Py}-\mathrm{N}_{-}-\mathrm{CH}_{2}\right)$, 39.0 (1C, Py-CH), 27.6 (2C, Py- $\left.\mathrm{CH}_{2}\right)$; LC-MS: 413.5 (Calculated mass for $+\mathrm{M}, 414.8)$; FT-IR $\left(\mathrm{cm}^{-1}, \mathrm{KBr}\right)$ : $3173.4(\mathrm{NH}), 2854.7(-\mathrm{CH}), 1659(\mathrm{C}=\mathrm{O}), 1586.7(\mathrm{C}=\mathrm{N})$; HPLC purity: $97.8 \%$; mp: $188.11^{\circ} \mathrm{C}-189.1^{\circ} \mathrm{C}$.

\subsection{Anti-Bacterial Assay}

All the synthesized hydrazones were tested for their anti-bacterial activity against a set of bacterial strains, namely, Staphylococcus aureus, and Pseudomonas aeruginosa by paper disc diffusion method with different concentrations of the solutions prepared in Dimethyl sulfoxide (DMSO). The reason of choosing DMSO for antibacterial studies was that it has no effect on the above mentioned bacterial strains. Nutrient agar was used as the culture medium for the growth of bacterial colony that was prepared by using peptone $(3.0 \mathrm{~g}), \mathrm{NaCl}$ $(3.0 \mathrm{~g})$, Yeast $(1.5 \mathrm{~g})$, Agar $(6.0 \mathrm{~g})$ in $300 \mathrm{~mL}$ of distilled water with $\mathrm{pH}$ at 7.0 The as prepared medium is autoclaved at 15 pa for 20 minutes and kept at $85^{\circ} \mathrm{C}$ for 30 minutes to sterilize the media. This media was then poured into petridishes slowly in laminar flow environment, allowed to solidify and kept at $30^{\circ} \mathrm{C}$ for $24 \mathrm{hrs}$. The bacterial strains were inoculated by spreading in peptidases and its temperature is maintained at $30^{\circ} \mathrm{C}$ for $24 \mathrm{hr}$. Using paper disc $(8 \mathrm{~mm})$ in nutrient agar culture medium, different concentrations $(50,100,150,200,250$ $\mu \mathrm{g} / \mathrm{mL}$ ) of the newly synthesized hydrazones (10-13) were loaded through bacteria free micro pipettes. The anti-bacterial activity was determined by measuring the zone of inhibition in millimeters and compared with standard drug Ciprofloxacin and Cefaclor.

\section{Conclusions}

We have developed the simple and crucial synthetic technique of vanillin related hydrazone derivatives and the reactions occurred very fast, under mild condition using reasonable reagents and solvents, yield is also higher. The anti-bacterial activity of synthesized novel hydrazones were effectively screened against Gram positive $S$. aureus and Gram-negative P. aeruginosa bacterial strains. Most of these compounds show moderate antibacterial activity comparable with to marketable compounds. The zone of inhibition of tested compounds shows, the vanillin coupled hydrazone derivatives encompass potent bio-activities against bacterial strains. Due to the strong bio-activity of our synthesized hydrazones can be further allowed to attempt other bio-activities against a number of diseases and this work will be precious for further studies in terms of toxicity effect and Quantity Structural Activity Relationship (QSAR) to improve their biological and pharmacological properties.

\section{Acknowledgements}

We are appreciative to Dr. A. Thamaraichelvan and Dr. M.Ganesan, Department of Chemistry, Thiagarajar Col- 
lege, Madurai, India for giving constant support to this research work. Funding support from the DST project SR/ME/S-3/0016/2008 is also acknowledged.

\section{References}

[1] S. Riva and L. G. Silvestri, "Rifamycine: A General View," Annual Review of Microbiology, Vol. 26, 1972, pp. 199-224. doi:10.1146/annurev.mi.26.100172.001215

[2] V. I. Yudelevich, V. V. Belakhov, E. V. Komarov, B. I. Ionin, G. L. Myasnikova, M. S. Polyak, M. A. Shneider and L. A. Rachkovskaya, "Synthesis and Biological Activity of New Derivatives of 1,2,3,4-Tetrahydroquinazolin-4-one," Pharmaceutical Chemistry Journal, Vol. 18, No. 10, 1984, pp. 704-707. doi:10.1007/BF00773018

[3] S. Rollas and S. G. Kucukguzel, "Biological Activities of Hydrazone Derivatives," Molecules, Vol. 12, No. 8, 2007, pp. 1910-1939.

[4] O. A. Olayinka, A. O. Craig, C. N. Obinna and A. A. David, "Microwave Assisted Synthesis and Antimicrobial Activity of 2-Quinoxalinone-3-hydra-zone Derivatives," Bioorganic \& Medicinal Chemistry, Vol. 18, No. 1, 2010, pp. 214-221. doi:10.1016/j.bmc.2009.10.064

[5] S. Rollas, N. Gulerman and H. Edeniz, "Synthesis and Antimicrobial Activity of Some New Hydrazones of 4-Fluorobenzoic Acid Hydrazide and 3-Acetyl-2,5-disubstituted-1,3,4-oxadiazolines," IL Farmaco, Vol. 57, No. 2,. 2002, pp. 171-174.

[6] A. Cukurovali, B. Yilmaz, S. Gur and C. Kazaz, "Synthesis, Antibacterial and Antifungal Activity of Some New Thiazolylhydrazone Derivatives Containing 3-Substituted Cyclobutane Ring," European Journal of Medicinal Chemistry, Vol. 41, No. 2, 2006, pp. 201-207. doi:10.1016/j.ejmech.2005.01.013

[7] J. Capilla, C. Serena, F. Javier, T. Ortoneda and J. Guarro, "Efficacy of Voriconazole in Treatment of Systemic Scedosporiosis in Neutropenic Mice," Antimicrobial Agents Chemother, Vol. 47, No. 12, 2003, pp. 3976-3978. doi:10.1128/AAC.47.12.3976-3978.2003

[8] M. G. Mamolo, V. Falagiani, D. Zampieri, U. Vio, E. Banfi and G. Scialino, "Synthesis and Antimycobacterial Activity of (3,4-Diaryl-3H-thiazol-2-ylidene)-hydrazide Derivatives," IL Farmaco, Vol. 58, No. 9, 2003, pp. 631637.

[9] J. R. Dimmock, S. C. Vasishtha and J. P. Stables, “Anticonvulsant Properties of Various Acetylhydrazones, Oxamoylhydrazones and Semicarbazones Derived from Aromatic and Unsaturated Carbonyl Compounds," European Journal of Medicinal Chemistry, Vol. 35, No. 2, pp. 241248.

[10] P. C. Lima, L. M. Lima, K. C. Silva, P. H. Leda, A. L. P. Miranda, C. A. M. Fraga and E. J. Barreiro, "Synthesis and Analgesic Activity of Novel $\mathrm{N}$-Acylarylhydrazones and Isosters, Derived from Natural Safrole," European Journal of Medicinal Chemistry, Vol. 35, No. 2, 2000, pp. 187-203. doi:10.1016/S0223-5234(00)00120-3

[11] G. U. Salgin, K. N. Gokham, O. Gostal, Y. Koysal, E.
Kilici, S. Isik, G. Aktay and M. Ozalp, "1-Acylthiosemicarbazides, 1,2,4-Triazole-5(4H)-thiones, 1,3,4-Thiadiazoles and Hydrazones Containing 5-Methyl-2-Benzoxazolinones: Synthesis, Analgesic-Anti-Inflammatory and Antimicrobial Activities," Bioorganic \& Medicinal Chemistry, Vol. 15, No. 17, 2007, pp. 5738-5751. doi:10.1016/j.bmc.2007.06.006

[12] A. R. Todeschini, A. L. Miranda, C. M. Silva, S. C. Parrini and E. J. Barreiro, "Synthesis and Evaluation of Analgesic, Anti-Inflammatory and Antiplatelet Properties of New 2-Pyridylarylhydrazone Derivatives," European Journal of Medicinal Chemistry, Vol. 33, No. 3, 1998, pp. 189-199. doi:10.1016/S0223-5234(98)80008-1

[13] G. A. Silva, L. M. M. Costa, F. C. B. Brito, A. L. P Miranda, E. J. Barreiro and C. A. M. Fraga, "New Class of Potent Antinociceptive and Antiplatelet 10H-Phenothiazine-1-Acylhydrazone Derivatives," Bioorganic \& Medicinal Chemistry, Vol. 12, No. 12, 2004, pp. 31493158. doi:10.1016/i.bmc.2004.04.009

[14] A. Imramovsky, S. Polanc, J. Vinsova, M. Kocevar, J. Jampitek, Z. Reckova and J. A. Kaustova, "A New Modification of Anti-Tubercular Active Molecules," Bioorganic \& Medicinal Chemistry, Vol. 15, No. 7, 2007, pp. 2551-2559. doi:10.1016/j.bmc.2007.01.051

[15] Y. Janin, "Antituberculosis Drugs: Ten Years of Research," Bioorganic \& Medicinal Chemistry, Vol. 15, No. 7, 2007, pp. 2479-2513. doi:10.1016/j.bmc.2007.01.030

[16] L. C. du Toit, V. Pillay and M. P. Danckwerts, "Tuberculosis Chemotherapy: Current Drug Delivery Approaches," Respiratory Research, Vol. 7, 2006, p. 118. doi:10.1186/1465-9921-7-118

[17] L. Savini, L. Chiasserini, V. Travagli, C. Pellerano, E. Novellino, S. Consentino and M. B. Pisano, "New $\alpha-(N)$ Heterocyclichydrazones: Evaluation of Anticancer, AntiHIV and Antimicrobial Activity," European Journal of Medicinal Chemistry, Vol. 39, No. 2, 2004, pp. 113-122. doi:10.1016/j.ejmech.2003.09.012

[18] A. M. El-Hawash, W. A. E Abdel and M. A. El-Dewellawy, "Cyanoacetic Acid Hydrazones of 3-(and 4-)Acetylpyridine and Some Derived Ring Systems as Potential Antitumor and Anti-HCV Agents," Archiv der Pharmazie, Vol. 339, No. 1, 2006, pp. 14-23. doi:10.1002/ardp.200500161

[19] M. T. Cocco, C. Congiu, V. Lilliu and V. Onnis, "Synthesis and in Vitro Antitumoral Activity of New Hydrazinopyrimidine-5-carbonitrile Derivatives," Bioorganic \& Medicinal Chemistry, Vol. 14, No. 2, 2006, pp. 366-372. doi:10.1016/j.bmc.2005.08.012

[20] S. G. Kucukguzel, A. Mazi, F. Sahin, S. Ozturk and J. Stables, "Synthesis and Biological Activities of Diflunisal Hydrazide-Hydrazones," European Journal of Medicinal Chemistry, Vol. 38, No. 11-12, 2003, pp. 1005-1013. doi:10.1016/j.ejmech.2003.08.004

[21] V. Sridharan, P. T. Perumal, C. Avendano and J. C. Menendez, "The First Aza Diels-Alder Reaction Involving an $\alpha, \beta$-Unsaturated Hydrazone as the Dienophile: Stereoselective Synthesis of C-4 Functionalized 1,2,3,4Tetrahydroquinolines Containing a Quaternary Sterecen- 
ter," Organic \& Biomolecular Chemistry, Vol. 5, 2007, pp. 1351-1353. doi:10.1039/b703083e

[22] A. Espinel-Ingroff, "Standardized Disk Diffusion Method for Yeasts," Clinical Microbiology Newsletter, Vol. 29, No. 13, 2007, pp. 97-100. doi:10.1016/j.clinmicnews.2007.06.001

[23] W. L. F Armarego and C. L. L. Chai, "Purification of Laboratory Chemicals," 5th Edition, Elsevier, Amsterdam, 2003.
[24] A. R. Katritzky, H.-Y. He, Q. H. Long and A. L. Wilcoxb, "Preparation of 2,6-Dialkoxybenzaldehydes," ARKIVOC, Vol. 3, 2001, pp. 3-12.

[25] F. S. Pashkovskii, E. M. Shchukina, M. G. Gribovskii and F. A. Lakhvich, "Heterocyclic Analogs of Prostaglandins: IV. Synthesis of 3,7-Interphenylene 3,10(11)-dioxa-13azaprostanoids and 9-oxa-7-azaprostanoids Based on Tetronic Acid and Aromatic Aldehydes," Russian Journal of Organic Chemistry, Vol. 44, No. 5, 2008, pp. 657-670. doi:10.1134/S1070428008050047 